\title{
Caracterización de la capa formada por nitruración gaseosa del titanio a alta temperatura
}

\author{
D. RODRÍGUEZ, J.M. MANERO, F.J. GIL Y J.A. PLANELL \\ Centre de Recerca en Enginyeria Biomèdica (CREB); Dept. Ciència dels Materials i Enginyeria Metal-lúrgica \\ Escola Tècnica Superior d'Enginyeria Industrial de Barcelona; Universitat Politècnica de Catalunya; Av. Diagonal, 647; 08028 - Barcelona.
}

\begin{abstract}
El titanio y la aleación Ti6Al4V son materiales que presentan una excelente biocompatibilidad, si bien la falta de una buena resistencia al desgaste dificulta su uso en diversas aplicaciones biomédicas. Se ha estudiado el endurecimiento superficial del titanio por medio de la nitruración gaseosa con el fin de mejorar su resistencia al desgaste. Los resultados obtenidos muestran un gran incremento en la dureza superficial, de un 300\% con relación a las muestras no tratadas. Los datos de las difracciones de rayos $\mathrm{X}$ de la superficie de las muestras tratadas muestran la existencia de nitruros de titanio, principalmente nitruros- $\varepsilon\left(\mathrm{Ti}_{2} \mathrm{~N}\right)$, si bien también fueron detectados nitruros- $\delta(\mathrm{TiN})$. La difracción de electrones realizada en microscopio electrónico de transmisión (TEM) de regiones subsuperficiales de las muestras tratadas también detectaron la existencia de nitruros- $\varepsilon$. Observaciones con TEM de zonas a distancias de la superficie superiores a $5 \mu \mathrm{m}$ no revelaron la existencia de nitruros. Por tanto, los resultados obtenidos indican que el tratamiento de nitruración gaseosa podría ser usado para reducir el desgaste del titanio en aplicaciones biomédicas con cargas poco elevadas.
\end{abstract}

Palabras clave: titanio, nitruración, tratamiento superficial, endurecimiento superficial, nitruro de titanio

Characterisation of the layer by nitriding of titanium at high temperature

Titanium and Ti6Al4V alloy are biocompatible materials. However, their lack of good wear resistance limits their use for some biomedical applications. Surface hardening by nitriding has been studied in order to improve wear resistance of titanium. The results showed an increase of surface hardness, over 300\% when compared to non-treated samples. X-ray diffraction data of the surface of the treated samples showed the existence of titanium nitrides, mainly $\varepsilon$-nitrides $\left(\mathrm{Ti}_{2} \mathrm{~N}\right)$, although $\delta$-nitrides (TiN) were also detected. Selected area diffraction pattern in transmission electron microscope (TEM) of nearsurface regions detected the existence of $\varepsilon$-nitrides. TEM observations of regions at depths over $5 \mu \mathrm{m}$ did not registered any nitride. Therefore, the results suggest that the studied treatment could be used to reduce wear in biomedical applications with light and medium loads.

Keywords: titanium, nitriding, surface treatment, surface hardness, titanium nitride

\section{INTRODUCCIÓN}

El titanio y sus aleaciones son materiales de uso cada vez más extendido en el campo de los biomateriales (1). Esto se debe a su excelente biocompatibilidad, su bajo módulo de elasticidad y su baja densidad, que los convierten en uno de los metales con un comportamiento mecánico más semejante al del hueso (2). El titanio comercialmente puro (cp), por ejemplo, es el material de uso para la fabricación de implantes dentales. Sin embargo, a pesar de dichas ventajas, su uso en aplicaciones con existencia de fricción como componentes articulares es limitado, debido a su baja dureza y malas propiedades tribológicas (3).

Actualmente existen diversos tratamientos superficiales que intentan solucionar dichos problemas (4). Algunos de ellos, como los procesos termoquímicos, la deposición física en fase vapor (PVD), la deposición química en fase vapor (CVD), la implantación iónica y los tratamientos por láser producen resultados relevantes. Los más usados en el caso del titanio cp son la implantación iónica y la deposición física por plasma. En la implantación iónica se aceleran iones de nitrógeno, carbono u oxígeno mediante un potencial eléctrico de 10 a $30 \mathrm{kV}$ (5). Los iones acelerados impactan en la muestra y penetran en ella hasta $100 \mathrm{~nm}$, formando una capa enriquecida en la superficie del material con una elevada dureza debida a la formación de compuestos. En el proceso de implantación de titanio con iones nitrógeno, se forman nitruros de titanio, especialmente nitruros- $\delta(\mathrm{TiN})$, con un espesor de capa de hasta 100 nm y una dureza de hasta $1800 \mathrm{HV}$ (6).

Los tratamientos termoquímicos implican la introducción de carbono, oxígeno, nitrógeno y otros elementos intersticiales en el titanio por difusión en medio gaseoso (7). Se forma una capa superficial endurecida de un espesor de 25-100 $\mu \mathrm{m}$. Sin embargo, estos procesos termoquímicos han tenido un uso limitado debido a la fragilidad causada por el oxígeno y a la incorporación de hidrógeno a la estructura del titanio, a causa de las impurezas del medio gaseoso usado. Un desarrollo más reciente de la técnica es el tratamiento por plasma, donde se utiliza amoníaco o nitrógeno como medio para crear el plasma mediante la aplicación de un potencial eléctrico. Este plasma impacta en la superficie del material a tratar a temperaturas de $400-700^{\circ} \mathrm{C}$, limpiando la superficie y facilitando la difusión del nitrógeno en el titanio. Se forma entonces una capa de nitruros de titanio y de compuestos intermetálicos de hasta $10 \mu \mathrm{m}$ de 
espesor, con durezas superficiales de hasta 1500HV (8).

Todos los tratamientos citados permiten obtener importantes incrementos en la dureza superficial del titanio. Cuando se usa nitrógeno, el incremento de dureza se debe a la formación de nitruros de titanio, como el nitruro- $\delta(\mathrm{TiN})$ y el nitruro- $\varepsilon$ $\left(\mathrm{Ti}_{2} \mathrm{~N}\right)$, con contenidos de nitrógeno superiores al 33\%, como puede observarse en el diagrama de fases Ti-N (Figura 1) (9).

El tratamiento de endurecimiento superficial estudiado en este trabajo es el tratamiento termoquímico de difusión gaseosa de nitrógeno a alta temperatura. Si bien la literatura indica la existencia de algunos problemas relacionados con este tratamiento, como la necesidad de largos tiempos de tratamiento (superiores a 16 horas) (7), y elevadas temperaturas de tratamiento (por encima de $1000^{\circ} \mathrm{C}$ ) (10), nuevos estudios de este proceso experimental muestran que, para ciertas aplicaciones como son los implantes, este proceso podría ser útil incluso a tiempos y temperaturas de tratamiento más cortos y bajas (11). El nitrógeno se difunde en el titanio formando una capa de titanio de fase $\alpha$, enriquecida con nitrógeno de 20-200 $\mu \mathrm{m}$ de espesor. El grosor de la capa, la dureza superficial y la resistencia al desgaste dependen de la temperatura y tiempo de tratamiento. En este trabajo se ha estudiado la formación de nitruros mediante este tratamiento, sus características y el grosor de la capa de nitruros formada.

\section{PROCEDIMIENTO EXPERIMENTAL}

\subsection{Preparación de las muestras}

El material estudiado es titanio comercialmente puro (cp) de grado 2, con una dureza media en recepción de $283 \mathrm{HV}$. Se prepararon 4 discos de titanio de $13 \mathrm{~mm}$ de diámetro y $3 \mathrm{~mm}$ de grosor, pulidos mediante alúmina en suspensión hasta obtener una rugosidad superficial de $\mathrm{R}_{\mathrm{a}}<0,05 \mu \mathrm{m}$. Las muestras se trataron a una temperatura de 800 y $850^{\circ} \mathrm{C}$, durante 1 y 4 horas en ambos casos. Como control se utilizó un disco no tratado. Para el tratamiento térmico se empleó un horno con una atmósfera controlada de nitrógeno de alta pureza.

\subsection{Caracterización de la dureza superficial}

Se realizaron medidas de microdureza según la escala Vickers (HV) en un microdurómetro marca Matzusawa, aplicando una carga de 100 gramos $\left(\mathrm{HV}_{0,1}\right)$ durante 15 segundos. Se realizaron medidas en la superficie de la muestra, con cálculo de la media de cinco medidas.

\subsection{Caracterización mediante microscopía electrónica de transmisión}

A fin de caracterizar la capa formada en la superficie del titanio, se utilizó la difracción de electrones de selección de área (selected area diffraction pattern). Ésta se realizó en un microscopio electrónico de transmisión JEOL 1200XL, con un potencial de $120 \mathrm{kV}$, utilizando una constante de cámara de 80 $\mathrm{cm}$. El adelgazamiento final de las muestras se realizó mediante ion milling en un equipo PIDS Gatan, utilizando una tensión de $5 \mathrm{kV}$ y un ángulo de $8^{\circ}$, obteniéndose zonas de observación en un punto situado a 2-4 $\mu \mathrm{m}$ de la superficie tratada.

\subsection{Caracterización mediante difracción de rayos $X$}

El estudio mediante difracción de rayos $\mathrm{X}$ se realizó en un difractómetro Siemens D500 controlado por ordenador, con

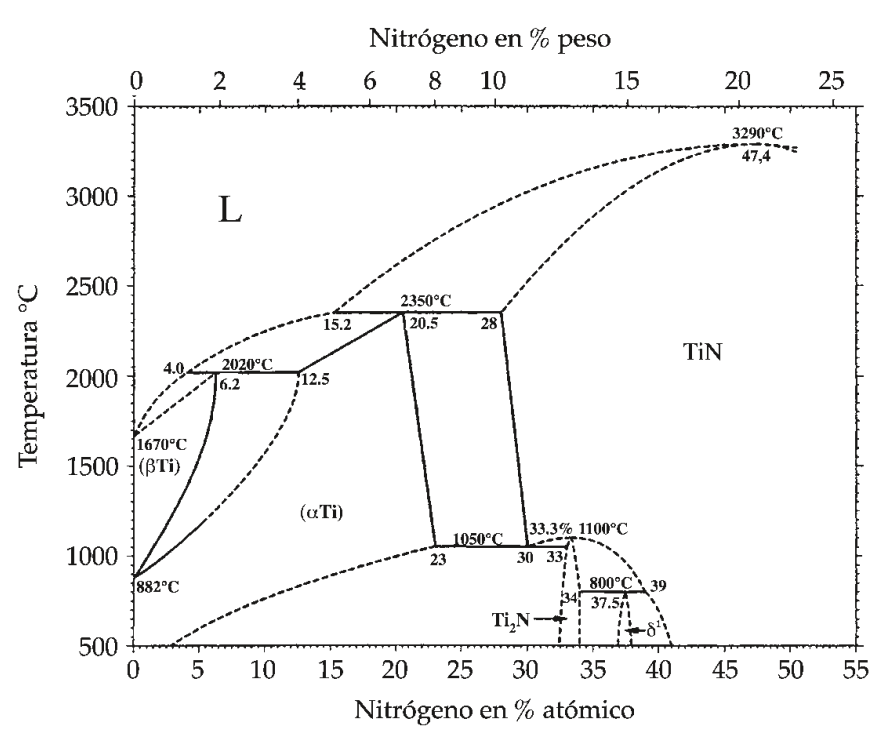

Figura 1. Diagrama de fases del sistema Ti-N (9).

filtro de níquel para la radiación $\mathrm{K}_{\mathrm{a}}$ del cobre y monocromador de grafito. Las condiciones de trabajo fueron una diferencia de potencial de $40 \mathrm{kV}$ y una intensidad de corriente de $30 \mathrm{~mA}$, con un tiempo de integración de 3 segundos a intervalos de $0,05^{\circ}(2 \theta)$ en modo continuo, desde $9,974^{\circ}$ a $100^{\circ}(2 \theta)$.

\subsection{Medición del espesor de la capa superficial obtenida}

El espesor de la capa obtenida se midió mediante análisis de imagen de fotografías obtenidas en un microscopio electrónico de barrido. Las muestras fueron cortadas en sección transversal, embebidas en una resina conductora para evitar fenómenos de corte, y pulidas con papel de $\mathrm{SiC}$ y suspensión de alúmina, hasta obtener una superficie especular. Las superficies pulidas fueron atacadas con un reactivo $\left(10 \% \mathrm{HNO}_{3}, 5 \% \mathrm{HF}\right.$, bal. $\left.\mathrm{H}_{2} \mathrm{O}\right)$ a fin de revelar la microestructura. Tras el ataque químico, las muestras fueron observadas en un microscopio electrónico de barrido JEOL JSM-6400.

\section{RESULTADOS}

\subsection{Dureza de la capa obtenida}

Las medidas de microdureza tomadas en la superficie de las muestras indicaron la existencia de incrementos de dureza de más del 300\% (superiores a $1000 \mathrm{HV}_{0.1}$ ) con relación a las muestras no tratadas, con dureza media de 283 HV (Tabla I). Los valores obtenidos presentan una relación proporcional con la temperatura y tiempo de tratamiento.

TABLA I. MicrodurEZAS SUPERFICIALES DE LAS MUESTRAS DE TITANIO NITRURADAS.

\begin{tabular}{cll} 
& \multicolumn{2}{c}{ Dureza $\left[H V_{0.1}\right]$} \\
\cline { 2 - 3 } & $1 \mathrm{~h}$ & $4 \mathrm{~h}$ \\
\hline $\mathbf{8 0 0 ^ { \circ } \mathrm { C }}$ & 420 & 621 \\
$\mathbf{8 5 0 ^ { \circ } \mathbf { C }}$ & 626 & 1012 \\
\hline En recepción & $\mathbf{2 8 3}$
\end{tabular}




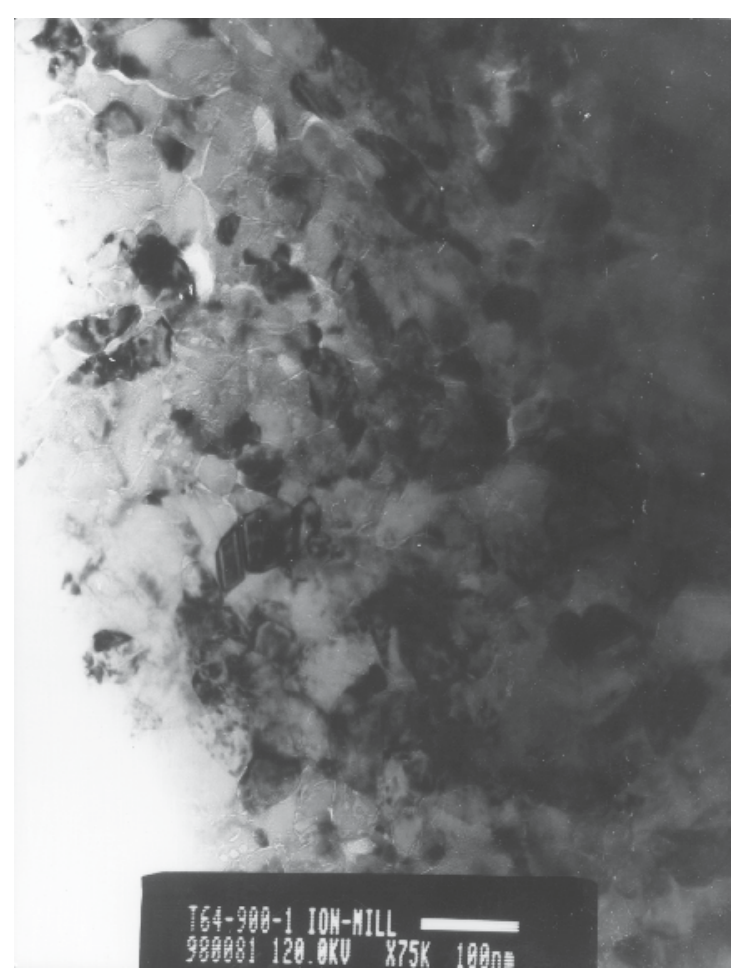

Figura 2. Microestructura de la capa nitrurada.

\subsection{Caracterización mediante microscopía electrónica de transmisión}

La observación mediante microscopía electrónica de transmisión mostró la existencia de nitruros de titanio, en forma de minúsculos granos con un diámetro igual o inferior a $200 \mathrm{~nm}$ (Figura 2). La indexación de los diagramas de difracción obtenidos sobre dichos nitruros (Figura 3) confirmó la existencia de un nitruro- $\varepsilon\left(\mathrm{Ti}_{2} \mathrm{~N}\right)$ tetragonal con parámetros de red $\mathrm{a}=0,468$ $\mathrm{nm}$ y $\mathrm{c}=0,295 \mathrm{~nm}$, muy similares a los valores dados por la bibliografía, de a =0,4943 nm y c =0,3036 nm [12].

\subsection{Caracterización mediante difracción de rayos $X$}

Los resultados de los estudios de difracción de rayos $\mathrm{X}$ indicaron, para todas las muestras, la existencia de una capa de nitruros de titanio formada por titanio- $\varepsilon\left(\mathrm{Ti}_{2} \mathrm{~N}\right)$ y mostraron también la presencia de titanio- $\delta(\mathrm{TiN})$, si bien en una proporción menor (Figura 4).

El desplazamiento de los picos de difracción correspondientes al titanio- $\alpha$ hacia ángulos más pequeños es indicio de la incorporación del nitrógeno a la microestructura hexagonal compacta del titanio como intersticial, ya que dicho desplazamiento es debido a ligeras modificaciones que se producen en los parámetros de red debidos a la distorsión inducida por el átomo de nitrógeno (Figura 5).

\subsection{Medición del espesor de la capa superficial obtenida}

El espesor de las capas obtenidas fue del orden de los 2-4 $\mu \mathrm{m}$ (figura 6), como cabría esperar a partir del hecho de que en los espectros de difracción de rayos $\mathrm{X}$ de las muestras tratadas siguieran apareciendo los picos de difracción correspondientes al titanio- $\alpha$. La variación del espesor de las capas es peque-

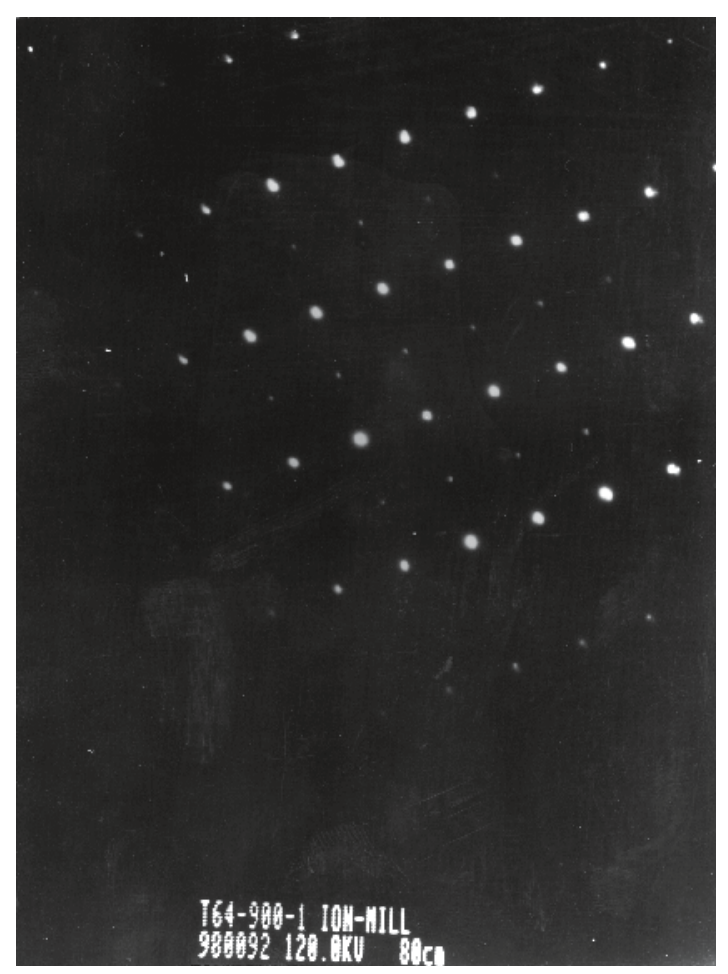

Figura 3. Difracción de electrones del $\mathrm{Ti}_{2} \mathrm{~N}$, con eje de zona [001]

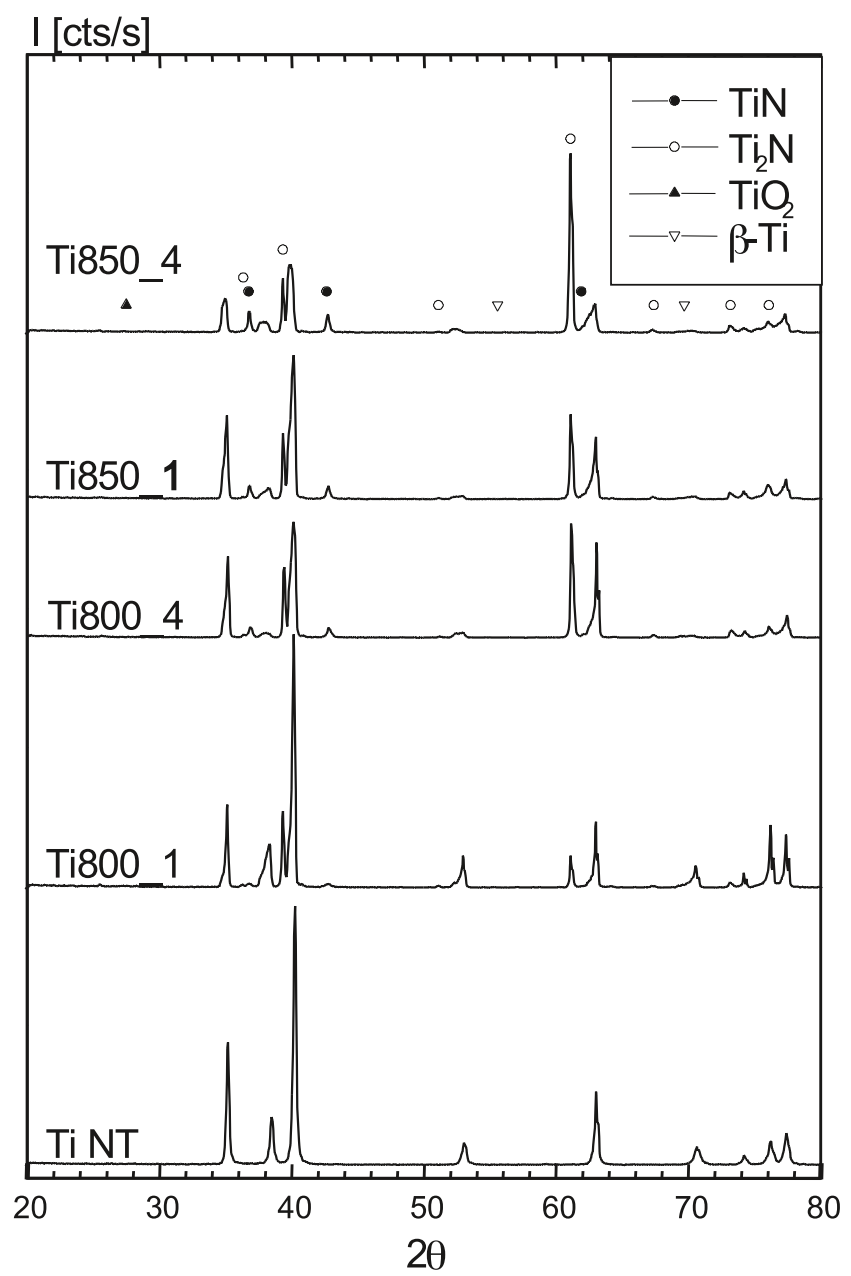

Figura 4. DRX titanio y titanio tratado. 
ña a $800^{\circ} \mathrm{C}$, si bien a $850^{\circ} \mathrm{C}$ el espesor de la capa se duplicó al aumentar el tiempo de tratamiento de una a cuatro horas, tal y como se muestra en la Tabla II.

\section{DISCUSIÓN}

A diferencia de los tratamientos de nitruración gaseosa para el acero, en los que se usa amoníaco como portador, en el caso del titanio no se puede seguir el mismo procedimiento debido al efecto de fragilización producido por el hidrógeno. El uso de nitrógeno puro parece ser suficiente para generar una capa superficial nitrurada, aumentando la dureza superficial del titanio en más de un $300 \%$, aún para tratamientos térmicos de duración inferior a 4 horas. El incremento de dureza se debe tanto a la capa superficial de nitruros, como a la capa subsuperficial de titanio enriquecido con nitrógeno intersticial. La deformación de la microestructura producida por el nitrógeno genera un incremento de la dureza del material modificado, que se refleja en el incremento de dureza obtenida.

La existencia de nitruros titanio- $\varepsilon\left(\mathrm{Ti}_{2} \mathrm{~N}\right)$ y titanio- $\delta(\mathrm{TiN})$ es coherente con el diagrama de fases Ti-N [7], el cual presenta la formación de $\mathrm{Ti}_{2} \mathrm{~N}$ para concentraciones de nitrógeno del 33 at $\%$, y de TiN para valores del 33 al 55 at $\%$. De hecho, la poca cantidad de nitruro titanio- $\delta$ es señal de que la cinética de difusión dificulta obtener concentraciones locales de nitrógeno superiores al 33 at $\%$.

La poca variación del espesor de las capas nitruradas puede ser debido a un efecto cinético de barrera, debido a la acumulación superficial de nitruros de titanio. La difusión del nitrógeno en los nitruros es menor que en titanio puro, de forma que la capa superficial de nitruros actúa como una barrera a la incorporación de más nitrógeno, lo que dificulta el crecimiento del espesor de la capa mediante incrementos de la temperatura y tiempo de tratamiento.

La posibilidad de obtener capas de nitruros mediante este procedimiento amplia el campo de posibilidades de endurecimiento superficial del titanio a bajo coste para aplicaciones biomédicas.

\section{AGRADECIMIENTOS}

Los autores desean agradecer a Technalloy S.A. la amable donación del material usado en este estudio, así como a Yves Maniette, de los Servicios Científico-Técnicos de la Universidad de Barcelona por su colaboración en la preparación de las muestras. Este trabajo ha sido financiado por el proyecto de la CICYT MAT98-0415.

\section{BIBLIOGRAFÍA}

1. S.A. Brown, J.E. Lemons, "Medical Applications of Titanium and its Alloys" ASTM STP 1272, Ann Harbor, USA, 1996

2. M. Donachie, "Titanium and Titanium Alloys" ASM, Ohio, USA, 1987.

3. M.A. Khan, R.L. Williams, D.F. Williams, "In-Vitro Corrosion And Wear Of Titanium Alloys In The Biological Environment" Biomaterials, 17, pp. 21172126 (1996).

4. T. Bell, H.W. Bergmann, J. Lanagan et al, "Surface Engineering Of Titanium With Nitrogen" Surface Engineering, 2, pp. 133-143 (1986)

5. T. Röstlund, B. Albrektsson, T. Albrektsson, H. Mckellop, "Wear of ionimplanted pure titanium against UHMWPE”, Biomaterials, 10, pp. 176-181 (1989)

6. J.P. Blanchard, A. Chen, Q. Bogin, “The Relationship Between Depth Profiles Of
TABLA II. ESPESOR DE LAS CAPAS OBTENIDAS POR NITRURACIÓN.

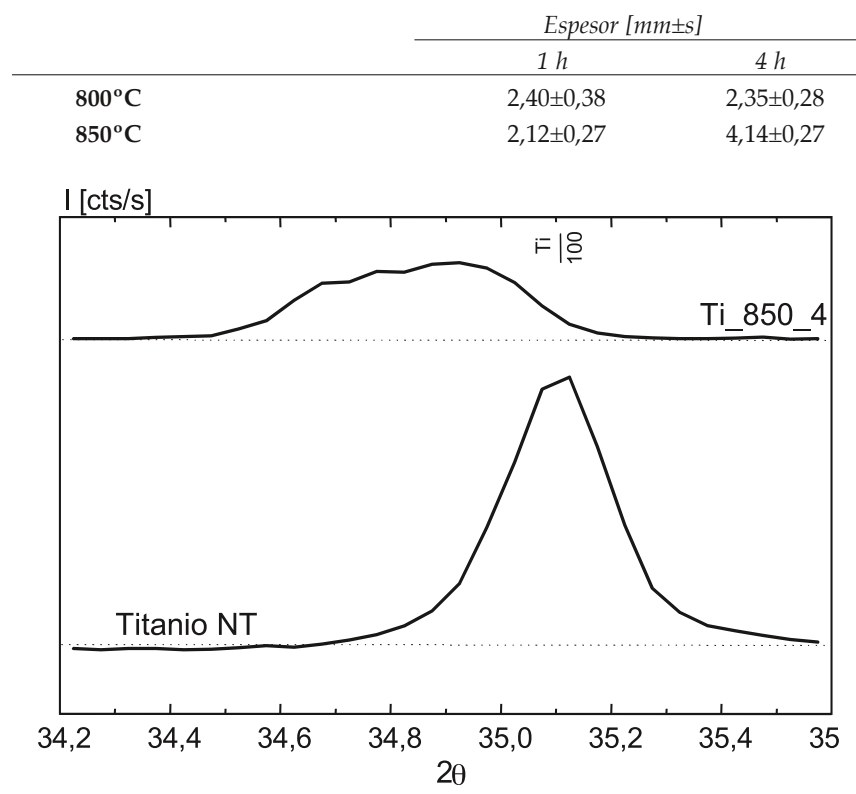

Figura 5. Desplazamiento del pico del titanio [100] causado por el nitrógeno.

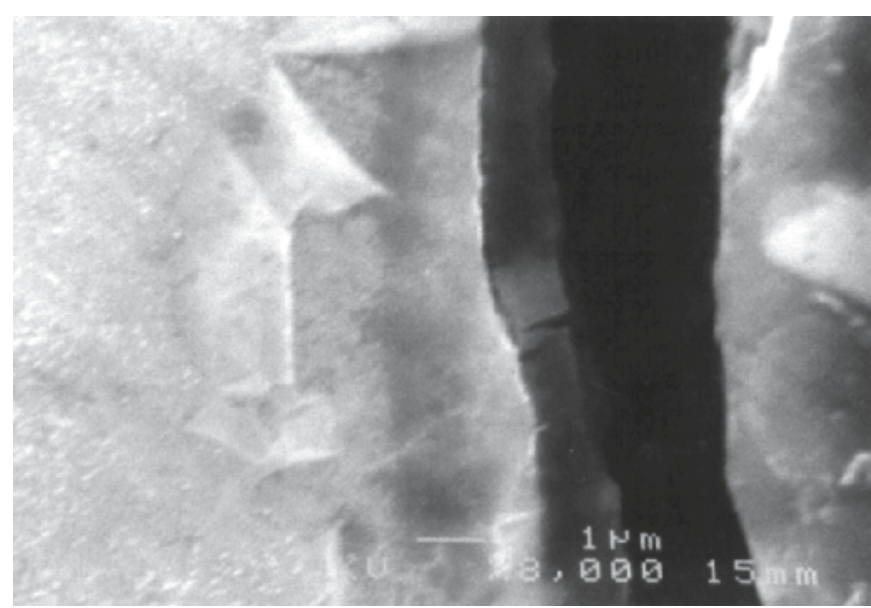

Figura 6. Capa nitrurada en sección transversal.

Nitrogen Concentration, Hardness, And Wear Rate In Ion-Implanted Ti-6Al-4V", Nuclear Instruments And Methods In Physics Research, 82, pp. 63-68 (1993).

7. J.L. Wyatt, N.J. Grant, "Nitriding of titanium with ammonia", Transactions ASM, 46, pp. 540-567 (1954)

8. J. Lanagan, "Properties Of Plasma Nitrided Titanium Alloys", Proc. $6^{\text {th }}$ World Conference On Titanium, Cannes, France, pp. 1957-1962 (1988).

9. H.A. Wriedt, J.L. Murray, “The N-Ti system" Bull alloy phase diagrams, 8, pp. 378-388 (1987).

10. R.W. Hanzel, "Surface Hardening Processes for Titanium and its Alloys", Metal Progress, 3, pp. 89-96 (1954).

11. Caracterización de la capa formada por nitruración gaseosa del titanio a alta temperatura. Rodríguez, J.M. Manero, F.J. Gil. 5th World Biomaterials Congress, v. II, Ed. Canadian Biomaterials Society, pp. 477 (1996).

12. B. Holmberg, "Structural Studies On The Titanium-Nitrogen System" Acta Chem Scand, 16, pp. 1255-1261 (1962). 